\title{
Role of Kaletra (combination of lopinavir and ritonavir) in the treatment of COVID-19 virus infection; an issue related to kidney transplant patients - A review study on current knowledge
}

\author{
Seyed Seifollah Beladi Mousavi ${ }^{\circledR}$, Fatemeh Hayati, Ehsan Valavi ${ }^{\circledR}$, Isa Rezaee ${ }^{\circledR}$, Shokouh Shayanpour ${ }^{\circledR}$, \\ Leila Sabetnia ${ }^{*} \mathbb{D}$
}

Chronic Renal Failure Research Center, Ahvaz Jundishapur University of Medical Sciences, Ahvaz, Iran

Correspondence to:

Leila Sabetnia, Email:

sabetnia-|@ajums.ac.ir

Received: 9 Sep. 2020

Accepted: 4 Dec. 2020

ePublished: 29 Dec. 2020

Keywords: Kaletra, Lopinavir and ritonavir, COVID-19, Kidney transplant patients, SARS-CoV-2

\begin{abstract}
A number of therapies are prescribed for the treatment of COVID-19, but none of them have proven efficacy. In this review article, we summarized the pharmacodynamic and pharmacokinetic properties, effect and potential toxicity of Kaletra (combination of lopinavir and ritonavir) among kidney transplant (KTP) patients who have COVID-19. We used a variety of sources by searching through PubMed, Scopus, Embase and Current Content to collect current data about our issue. Articles published in the English language, as full-text manuscripts, and or as abstract form were included in the study. Lopinavir and ritonavir are two structurally related novel protease inhibitors which have antiretroviral properties. They have primarily been used as part of combination therapy for the treatment of HIV, SARS-CoV and MERS-CoV viruses. However, it seems that use of Kaletra is not associated with clinical improvement, or reduces mortality among patients including KTP recipients who have laboratory-confirmed COVID-19. On the other hand, Co-administration of Kaletra with medications that are commonly used among KTP recipients including calcineurin inhibitors and mTOR inhibitors has profound drug-drug interactions. Co-administration of Kaletra with these medications could lead to significant and unexpected increase of blood concentrations of both calcineurin and mTOR inhibitors and therefore frequent monitoring of the immunosuppressive drugs concentrations are necessary to optimize immunosuppressive therapy and prevention of toxicity. It is important to note that frequent monitoring of the immunosuppressive drugs concentration is expensive and also not easily available in many countries including our country. According to the above important points, we recommend that use of Kaletra among KTP patients who have laboratory-confirmed COVID-19 should be avoided especially among patients who are still on calcineurin and mTOR inhibitors.
\end{abstract}

\section{Introduction}

Novel coronavirusSARS-CoV-2 (COVID-19) is a newly discovered contagious disease. At the end of 2019, it was identified as the cause of a cluster of pneumonia in Wuhan of China and rapidly spread in almost all other countries. It is reported that globally, more than ten million peoples have laboratoryconfirmed SARS-CoV-2 throughout the world.

Fortunately most of the patients with COVID-19 have a mild disease or asymptomatic infection. However severe acute respiratory syndrome with more than 50 percent lung involvement on imaging within 24 to 48 hours, severe hypoxemia and dyspnea is also reported among significant percent of patients.

Although it is reported that severe illness can occur in otherwise healthy individuals, it predominantly occurs in adults who have underlying medical co-morbidities including cardiovascular disease, diabetes mellitus, hypertension, chronic lung disease, cancer (in particular hematologic malignancies, lung cancer, and metastatic disease), chronic kidney disease, obesity and smoking.

Solid organ transplant recipients including kidney transplant (KTP) patients may also be at increased risk for COVID-19.

Like immunocompetent hosts without kidney disease, some KTP patients with known or suspected COVID-19-19 have mild diseases that are managed in the outpatient setting. Unfortunately there is little evidence among the treatment of COVID-19 in KTP patients with moderate to severe disease who admitted to the hospital.

A number of therapies are prescribed for the treatment of COVID-19, however none of them have proven efficacy, there are concerns about their potential toxicity 


\begin{abstract}
Key point
Some clinical trials performed among patients with confirmed SARS-CoV-2 infection to evaluate the effect of Kaletra among these patients. However the results of these studies were not promising. On the other hand, coadministration of Kaletra with medications which are commonly used among KTP recipients including calcineurin inhibitors and mTOR inhibitors has profound drug-drug interactions and lead to significant and unexpected increase of blood concentrations of both calcineurin and mTOR inhibitors. Therefore we recommend that the administration of Kaletra among KTP patients who have SARS-CoV-2 should be avoided especially among patients who are still on calcineurin and mTOR inhibitors.
\end{abstract}

and therefore they are not recommended to use among patients who have mild disease outside of a clinical trial (1). However among patients with moderate to severe disease who admitted to the hospital, many agents with known or putative antiviral or immunomodulating effects have been proposed including Kaletra (combination of lopinavir and ritonavir), remdesivir, favipiravir, interferon beta and hydroxychloroquine/chloroquine (1-3).

In this review article, we summarized the pharmacodynamic and pharmacokinetic properties, effect and potential toxicity of Kaletra among KTP patients with known or suspected COVID-19-19.

\section{Materials and Methods}

For this review article, we used a variety of sources by searching through PubMed, Scopus, Embase and Current Content to collect current data about our issue. The search was performed by using several predefined combinations of the following keywords and or their equivalents: 'SARSCoV-2', 'COVID-19', lopinavir and ritonavir,' 'Kaletra', and 'kidney or solid organ transplantation'. Articles published in the English language, as full-text manuscripts, and or as abstract form were included in the study.

\section{Pharmacodynamic and pharmacokinetic properties}

Lopinavir and ritonavir are two structurally related novel aspartate protease inhibitors (PI) which have antiretroviral properties. They have primarily been used as part of combination therapy (branded as Kaletra or Aluvia) for the treatment and prevention of human immunodeficiency virus-1 (HIV-1) infection (4-6).

Lopinavir is a highly potent and selective protease inhibitor and it has an approximately ten fold higher in vitro PI activity than ritonavir among HIV patients, however in vivo PI activity of lopinavir is significantly decreased by hepatic metabolism through cytochrome P450 3A isoenzyme (5-7).

It has been reported that co-administration of lopinavir with low-dose of ritonavir inhibits metabolic inactivation of lopinavir through the inhibition of cytochrome P450CYP3A4and significantly improves plasma half life, pharmacokinetic properties and antiretroviral activity of Lopinavir among HIV patients.

In addition, according to available good randomized clinical trials, the rate of resistance to lopinavir is significantly decreased among HIV patients who take lopinavir with low-dose of ritonavir (4-7). Therefore coformulated lopinavir/ritonavir as part of combination therapy has produced in recent years by some companies for ease of administration and also to ensure both drugs are taken together by HIV patients.

In overall Kaletra is well tolerated among adult patients however few adverse events may occurr among users. Diarrhea, nausea, vomiting and other gastrointestinal disturbances, asthenia, headache, weakness, drowsiness, dizziness, bad taste in the mouth and skin rash are the most common side effects contributed to Kaletra in adult patients (4-6).

The rationale for use of Kaletra in COVID-19

As we know, there is not any pharmacological therapy with proven efficacy for COVID-19. On the other hand, the development of new and effective drugs for an infection will require extensive preclinical testing followed by double-blind and randomized clinical trials. Therefore some of investigators re-purposed currently available drugs which are effective against other coronaviruses for immediate use among these patients $(6,7)$.

After the emergence of SARS in 2003, it was seen that Kaletra has some in vitro activity against the SARS-CoV which causes severe acute respiratory syndrome (SARS), and MERS-CoV which causes Middle East respiratory syndrome (MERS). Therefore it is purposed that this co-formulated medicine may reduce the risk of adverse clinical outcomes as well as viral load among patients who have been infected with these viruses $(4,5)$.

As an example, Chu et al in an open-label study in 2004, evaluated the effect of ribavirin and lopinavir/ ritonavir combination (400 $\mathrm{mg}$ and $100 \mathrm{mg}$, respectively) (treatment group) with ribavirin alone (control group) among patients with SARS.

The outcome of the patients was better and the viral load was significantly lower among the Kaletra group compared to the control group. In addition, the adverse outcome was also significantly lower in the treatment group than in the control group (4).

Chan et al also assessed the effect of Kaletra among a nonhuman primate model of common marmoset. They showed that the Kaletra-treated animals have better outcome with improved clinical, radiological and pathological findings than untreated animals. In addition, the mean viral loads in necropsied lung were also lower among Kaletra-treated animals compared to untreated animals (5).

According to the results of several studies, some randomized clinical trials performed to evaluate the effect of Kaletra among patients with confirmed SARS-CoV-2 infection. However the results of these studies were not promising $(6,7)$. As an example, the result of the study by Cao et al showed that use of Kaletra (lopinavir $400 \mathrm{mg}$, and 
ritonavir $100 \mathrm{mg}$ ) twice a day for 14 days among hospitalized adult patients with severe COVID-19 is not significantly accelerated clinical improvement (6). In this study which was a randomized, controlled, open-label clinical trial, 199 patients with serious COVID-19 infection randomly assigned in a 1:1 ratio to receive either Kaletra in addition to standard supportive care, or standard supportive care alone. Cao et al concluded that Kaletra treatment is not associated with clinical improvement, reduce mortality, or diminish throat viral RNA delectability among patients with laboratory-confirmed SARS-CoV-2 (6).

The result of ELACOI trial which was a single-blind randomized controlled trial in China is also similar to the study by Li et al. In this study, 44 patients with mean age of 49.4 which had mild or moderate clinical status were randomized to receive either lopinavir-ritonavir or umifenovir (another antiviral) and or standard care therapy without antiviral. This study showed, $38.1 \%$ of patients who received lopinavir-ritonavir deteriorated to severe/critical clinical status, compared to $12.5 \%$ and $14.3 \% 3$ in the umifenovir and control group respectively (7).

Cai et al in an observational study evaluated outcome of patients who received antiviral therapy including lopinavir/ritonavir or favipiravir (another antiviral agent) with those who did not receive antiviral therapy at a single hospital in China between January 11, and February 6, 2020. The results of the study showed that there is not any difference between patients who received or did not receive antiviral therapy in time to viral clearance (8).

In conclusion, according to the results of above and other available studies, it seems that the use of lopinavir/ritonavir is not associated with clinical improvement, or reduce mortality among patients with laboratory-confirmed SARS-CoV-2 and therefore there is not sufficient evidence to recommend it among these patients (6-8).

\section{COVID-19 among KTP patients}

End-stage renal disease is a worldwide public health problem with a high complication rate and kidney transplantation is the best option among these patients (9).

Although it is not known whether KTP patients are at higher risk for COVID-19 compare to the general population, it seems theoretically, that these patients may be at increased risk and poor outcomes due to high rates of comorbidities. In addition, solid organ transplant recipients including KTP patients are at higher risk for COVID-19 infection because they are immunocompromised and have frequent contact with the health care systems $(9,10)$.

Clinical features of COVID-19 among KTP recipients are variable and it may be similar to non-transplant patients. The most common presenting symptoms are fever, cough, and dyspnea, similar to the general population. However it seems that fever is less common as an initial symptom among KTP patients, possibly as a consequence of the effects of immunosuppressive therapy on the systemic inflammatory response (9-11). As an example, in case series of Akalin et al among $36 \mathrm{KTP}$ patients, fever was a presenting symptom in only 21 patients (58\%). Cough, dyspnea, myalgias and diarrhea was seen in 19, 16, 13 and 8 patients respectively.

On the other hand, lymphopenia is more common among KTP patients and may be more profound than non-transplant patients who have COVID-19 (10).

It is also suggested that organ transplant patients who have COVID-19 compared to non-immunocompromised patients may shed greater amounts of virus for longer durations. According to this issue, these patients should have a longer duration of isolation compared to nonimmunocompromised patients for reduction of spreading the infection to the general population (1-3).

\section{Lopinavir/ritonavir among KTP patients}

The management of solid organ transplant patients with COVID-19 infection including KTP patients is difficult however, similar to the general population, use of antiviral agents is generally limited to hospitalized patients who have or are at risk for severe disease, because none of these antiviral agents have proven efficacy and there are concerns about their potential toxicity.

Although according to the above studies, there is not sufficient evidence to recommend or suggest Kaletra among patients with laboratory-confirmed SARS-CoV-2, it is still used among these patients including solid organ transplant patients (1-3).

Unfortunately there is not any randomized and controlled clinical trial about the effect of Kaletra among KTP patients who have SARS-CoV-2 to confirm or exclude the possibility of a treatment benefit of this medicine. However according to the results of other studies that have evaluated the effect of Kaletra among immunocompetent hosts without kidney diseases, it seems that Kaletra had no or small beneficial effects among these patients (6-8). Additionally, concomitant use of Kaletra and medications which are commonly used among solid organ transplant recipients as the mainstays of immunosuppression regimens to prevent allograft rejection, may lead to complex drug-drug interactions (12-14).

Medications of immunosuppression regimens usually have narrow therapeutic ranges. Therefore, frequent monitoring of the drug concentration is necessary to optimize immunosuppression, especially among patients who are also taking medications like Kaletra, which have interactions with immunosuppression regimens $(15,16)$.

It is important to note that lower concentrations of immunosuppression regimens can induce organ transplant rejection and on the other hand, high concentrations of these medications can lead to toxicity aside from over immunosuppression- related complications, such as opportunistic infection $(15,16)$.

The calcineurin inhibitors including cyclosporine and tacrolimus bind with high affinity to a family of 
cytoplasmic proteins present in most cells and selectively inhibit calcineurin, a calcium- and calmodulin-dependent phosphatase, thereby impairing the transcription of interleukin (IL)-2 and several other cytokines in $\mathrm{T}$ lymphocytes including IL-3, IL-4, tumor necrosis factor-alpha, CD40L, granulocyte-macrophage colonystimulating factor, and interferon-gamma.

Both cyclosporine and tacrolimus are extensively metabolized by cytochrome P-450 3A4 (encoded by the CYP3A4 gene) in the liver, and to a lesser degree in the gastrointestinal tract, and the kidney. Therefore blood concentrations of both cyclosporine and tacrolimus are increased and decreased by inhibitors and inducers of P-450 3A4 respectively (11-16).

Ketoconazole, itraconazole, fluconazole, verapamil, diltiazem and erythromycin are known common inhibitors of P-450 3A4 and therefore increase blood concentrations of calcineurin inhibitors (13-15).

Recently, protease inhibitors are also known as potent inhibitors of CYP3A and profound interactions between calcineurin inhibitors with various protease inhibitors including Kaletra have been reported.

In addition both cyclosporine and tacrolimus are P-glycoprotein substrates and variations in expression and/or function of P-glycoprotein could also lead to accumulation of these drugs (13-16). Therefore, coadministration of protease inhibitors including Kaletra which are also inhibitors of P-glycoprotein, with calcineurin inhibitors could lead to increase in blood concentrations of both cyclosporine and tacrolimus.

As an example, Jain et al described a profound interaction between tacrolimus and Kaletra in three liver transplant HIV-positive patients. At the end of the study, they concluded that even very low-dose of tacrolimus as low as less than $1 \mathrm{mg} /$ week may be sufficient to maintain adequate blood tacrolimus concentrations among HIVpositive liver transplant patients who take Kaletra and further caution is necessary among patients who have hepatic dysfunction or are also on other inhibitors of P-450 3A4.

Several studies also postulated that Kaletra may almost completely inhibit CYP3A-mediated metabolism and P-glycoprotein-mediated efflux of tacrolimus among these patients (12-15).

In another study, Vogel et al described how dosing of cyclosporine can be adjusted to maintain effective immunosuppressive drug levels among three HIV-positive liver transplant patients who were on lopinavir-based or ritonavir-boosted indinavir antiretroviral therapy. They also showed a profound interaction between cyclosporine and protease inhibitor antiretroviral therapy and suggested that cyclosporine may be reduced to $5 \%$ of the standard dose to maintain blood cyclosporine concentrations within the target range among these patients (12).

Unfortunately we did not find a study to evaluate the interactions between Kaletra with newer immunosuppressive drugs like commercially available mammalian (mechanistic) target of rapamycin (mTOR) inhibitors including sirolimus, everolimus, and temsirolimus which are sometimes used for maintenance immunosuppressive therapy among solid organ transplant patients.

However, in recent years, an interaction between some protease inhibitors with sirolimus has been reported. Sirolimus, also known as rapamycin, is a P-glycoprotein substrate and it seems that variations in expression and/or function of P-glycoprotein may increase rapamycin levels. Several studies showed an interaction between sirolimus and nelfinavir, which is another protease inhibitor like Kaletra in a 40-year-old woman HIV-positive liver transplant patient $(11,13)$.

After normalizing the kinetic parameters to sirolimus dose of $1 \mathrm{mg} / \mathrm{d}$ among this patient who was on nelfinavir, 0 -hour trough sirolimus blood concentrations was ninefold and 24-hour trough sirolimus blood concentrations was five-fold higher compared with those who did not take nelfinavir (14-16).

According to the above study, it seems that concomitant use of all of protease inhibitors including Kaletra and mTOR inhibitors including sirolimus, everolimus, and temsirolimus, could lead to increase of blood concentrations of mTOR inhibitors and therefore may lead to complex drug-drug interactions (14-16).

\section{Conclusion}

In summary, the results of the above studies show that the use of Kaletra is not associated with clinical improvement among patients with SARS-CoV-2. Moreover, coadministration of lopinavir/ritonavir with medications which are commonly used among KTP recipients including calcineurin inhibitors like cyclosporine and tacrolimus and mTOR inhibitors like sirolimus and everolimus has profound drug-drug interactions. Co-administration of lopinavir/ritonavir with these medications could lead to a significant and unexpected increase of blood concentrations of both calcineurin and mTOR inhibitors. Therefore frequent monitoring of the immunosuppressive drug concentrations is necessary to optimize immunosuppressive therapy.

It is also important to note that frequent monitoring of the concentration of the immunosuppressive drug is expensive and also is not easily available in many countries including our country and therefore according to these important points, we recommend that the administration of Kaletra among KTP patients who have laboratoryconfirmed SARS-CoV-2 should be avoided especially among patients who are still on calcineurin and mTOR inhibitors.

Authors' contribution

SSBM, FH, and LS conducted literature review. EV and IR prepared 
the primary draft. SS and LS edited the paper. All authors read and signed the final paper.

\section{Conflicts of interest}

The authors declare that they have no competing interests.

Ethical considerations

Ethical issues (including plagiarism, data fabrication, double publication) have been completely observed by the authors.

\section{Funding/Support}

Chronic Renal Failure Research Center of Ahvaz Jundishapur University of Medical Sciences supported the study.

\section{References}

1. World Health Organization. Director-General's remarks at the media briefing on 2019-nCoV on 11 February 2020. https://www.who.int/dg/speeches/detail/who-director-generals-remarks-at-the-media-briefing-on-2019-ncov-on-11february-2020. Accessed February 12, 2020.

2. Centers for Disease Control and Prevention. 2019 Novel coronavirus, Wuhan, China. Information for Healthcare Professionals. https://www.cdc.gov/coronavirus/2019-nCoV/ hcp/index.html. Accessed February 14, 2020.

3. World Health Organization. Novel Coronavirus (2019nCoV) technical guidance. https://www.who.int/emergencies/ diseases/novel-coronavirus-2019/technical-guidance. Accessed February 14, 2020.

4. Chu CM, Cheng VCC, Hung IFN. Role of lopinavir/ritonavir in the treatment of SARS: Initial virological and clinical findings. Thorax. 2004;59:252-6. doi: 10.1136/thorax.2003.012658.

5. Chan JF, Yao Y, Yeung ML, Deng W, Bao L, Jia L. Treatment with lopinavir/ritonavir or interferon- $\beta 1 \mathrm{~b}$ improves outcome of MERS-CoV infection in a nonhuman primate model of common marmoset. J Infect Dis. 2015;212:1904-13. doi: 10.1093/infdis/jiv392.

6. Cao B, Wang Y, Wen D, Liu W, Fan JWG, Ruan L, et al. A trial of lopinavir-ritonavir in adults hospitalized with severe Covid-19. N Engl J Med. 2020;382:1787. doi: 10.7326/ACPJ202006160063.

7. Li Y, Xie Z, Lin W, Cai W, Wen C, Guan Y, et al. An exploratory randomized, controlled study on the efficacy and safety of lopinavir/ritonavir or arbidol treating adult patients hospitalized with mild/moderate COVID-19 (ELACOI). medRxiv. 2020. doi: 10.1016/j.medj.2020.04.001

8. Cai Q, Huang D, Ou P, Yu H, Zhu Z, Xia Z, et al. COVID-19 in a designated infectious diseases hospital outside Hubei Province, China. Allergy. 2020;75:1742-52. doi: 10.1111/ all.14309.

9. Pereira MR, Mohan S, Cohen DJ, Husain SA, Dube GK, Ratner LE, et al. COVID-19 in solid organ transplant recipients: Initial report from the US epicenter. Am J Transplant 2020;7:18001808. doi: 10.1111/ajt.15941.

10. Akalin E, Azzi $Y$, Bartash R, Seethamraju H, Parides $M$, Hemmige $V$, et al. Covid-19 and kidney transplantation. N Engl J Med 2020;382:2475-7. doi: 10.1056/NEJMc2011117.

11. Jain $A B$, Venkataramanan $R$, Eghtesad $B$, Marcos $A$, Ragni $M$, Shapiro $R$, et al. Effect of coadministeredlopinavir and ritonavir (Kaletra) on tacrolimus blood concentration in liver transplantation patients. Liver Transpl. 2003;9(9):954-60. doi: 10.1053/jlts.2003.50171.

12. Vogel M, Voigt E, Michaelis HC, Sudhop T, Wolff $M$, Türler $A$, et al. Management of drug-to-druginteractions between cyclosporine $\mathrm{A}$ and the protease-inhibitor lopinavir/ritonavir in liver-transplanted HIV-infected patients. Liver Transpl. 2004;10:939-44. doi: 10.1002/lt.20165.

13. Venkataramanan R, Fridell JA, Gadomski M, Shaw LM, Ragni $M$, et al. Nelfinavir, a protease inhibitor, increases sirolimus levels in a liver transplantation patient: a case report. Liver Transpl. 2002;8:838-40. doi: 10.1053/jlts.2002.34921.

14. Manez R, Martin M, Raman D, Silverman D, Jain A, Warty $\mathrm{V}$, et al. Fluconazole therapy in transplant recipients receiving FK506. Transplantation. 1994;57:1521-3. doi: 10.1097/00007890-199405270-00022.

15. Iwasaki $K$, Matsuda $H$, Nagase $K$, Shiraga T, Tokuma $Y$, UchidaK. Effects of twenty-three drugs on the metabolism of FK506 byhuman liver microsomes. Res Commun Chem Pathol Pharmacol. 1993;82:209-16.

16. Saeki T, Ueda K, Tanigawara Y, Hori R, Komano T. HumanPglycoprotein transports cyclosporin A and FK506. J Biol Chem. 1993;268:6077-6080. 